\title{
Fractionation of Sialylated Oligosaccharides, Glycopeptides, and Glycoproteins on Immobilized Elderberry (Sambucus nigra L.) Bark Lectin'
}

\author{
NAOTO SHIBUYA,* IRWIN J. GOLDSTEIN,* WILLEM F. BROEKAERT, $†$ \\ MAKUTA NSIMBA-LUBAKI, $\dagger$ BEN PEETERS, $\ddagger$ AND WILLY J. PEUMANS $\dagger$ \\ *Department of Biological Chemistry, University of Michigan, Ann Arbor, Michigan $48109 ; \dagger$ Laboratorium \\ voor Plantenbiochemie, Katholieke Universiteit Leuven, Kardinaal Mercierlaan 92, B-3030 Leuven \\ (Heverlee), Belgium; and $\ddagger$ Laboratorium voor Human Biologie, Katholieke Universiteit Leuven, \\ Universitair Ziekenhuis Gasthuisberg, Herestraat 49, B-3030 Leuven (Heverlee), Belgium
}

Received August 25, 1986, and in revised form November 5, 1986

A new plant lectin from elderberry (Sambucus nigra L.) bark, which was shown by immunochemical techniques to bind specifically to terminal Neu5Ac $(\alpha 2-6) \mathrm{Gal} / \mathrm{GalNAc}$ residues of glycoconjugates, was immobilized onto Sepharose 4B (SNA-Sepharose) and its carbohydrate binding properties was determined using a series of standard compounds. Oligosaccharides, glycopeptides, or glycoproteins containing terminal Neu5Ac $(\alpha 2-6) \mathrm{Gal} / \mathrm{GalNAc}$ sequences bound to SNA-Sepharose and were eluted with 50-100 mM lactose, whereas those with Neu5Ac $(\alpha 2-3) \mathrm{Gal} / \mathrm{GalNAc}$ failed to bind to this column. Furthermore, the SNA-Sepharose column was capable of resolving two oligosaccharides/glycopeptides based on the number of Neu5Ac $(\alpha 2-6) \mathrm{Gal}$ units present in each molecule. Application of this technique to two glycoproteins, fetuin and orosomucoid, revealed the presence of microheterogeneity. It was also shown that esterification of the carboxyl group of Neu5Ac units, or branching at the $O$-3 of the subterminal GalNAc (probably also Gal) destroyed the binding ability of the molecule. (C) 1987 Academic Press, Inc.

Sialylated glycoproteins, glycolipids, and oligosaccharides are widely distributed throughout the animal kingdom (1). These sialylated glycoconjugates function as cell surface receptors for bacteria, viruses, and mycoplasma (2-7), as antigenic immunodeterminants $(8,9)$, and also have been considered to serve as "anti-recognition markers" for the metabolic clearance system of serum components and of certain blood cells (10). Sialic acid is usually present as the nonreducing terminal sugar of carbohydrate chains, of ten linked to D-galactose $(\mathrm{Gal})^{2}$ or $N$-acetyl-D-galactosamine

\footnotetext{
1 This research was supported by National Institute of Health Grant GM 29470.

${ }^{2}$ Abbreviations used: SNA, Sambucus nigra $L$ agglutinin; Con A, concanavalin A; Gal, galactose; GalNAc, $N$-acetylgalactosamine; Neu5Ac, $N$-acetylneuraminic acid.
}

(GalNAc) residues through 2,3- or 2,6linkages. These sialylated glycoconjugates or their partial degradation products, glycopeptides and oligosaccharides, can be analyzed by the use of ion-exchange chromatography, a technique which resolves based on the density of sialic acid residues in the molecule $(11,12)$. However, using this approach it is very difficult to separate molecules which carry a similar number of sialic acid groups differing only in their linkage (i.e., 2,3- or 2,6-).

We recently discovered that a new plant lectin from elderberry (Sambucus nigra $L$.) bark (SNA) (13) specifically binds the Neu5Ac( $\alpha 2-6) \mathrm{Gal} / \mathrm{GalNAc}$ sequence (14). The affinity of SNA for oligosaccharides containing this Neu5Ac $(\alpha 2-6) \mathrm{Gal}$ sequence is 20-150 times greater than those with the Neu5Ac $(\alpha 2-3) \mathrm{Gal}$ sequence, and 1600 10,000 times greater than for Gal. Based 
on these results, we speculated that SNA would be a useful tool for the separation of sialylated glycoconjugates based on the type and number of Neu5Ac-Gal/GalNAc sequences in the molecule. This report demonstrates the application of immobilized SNA for the fractionation of glycoconjugates as well as affording further insight regarding the carbohydrate binding specificity of this unique lectin.

\section{MATERIALS AND METHODS}

SNA-Sepharose. SNA was purified as previously described (13) and coupled to Sepharose $4 B$ by the method of March et al. (15). Neu5Ac( $\alpha 2-6)$ lactose (final concentration, $0.2 \mathrm{mM}$ ) was added to the reaction mixture to protect the carbohydrate binding site. The final product contained approximately $1.2 \mathrm{mg} \mathrm{SNA} / \mathrm{ml}$ of the gel.

Oligosaccharides, glycopeptides, and glycoproteins. Purified Neu5Ac( $\alpha 2-3)$ lactose, Neu5Ac( $\alpha 2-6)$ lactose, and DSL were the gifts of Dr. V. Ginsburg of the National Institutes of Health. ${ }^{8}$ II-labeled milk oligosaccharides, LSTa, LSTb, and LSTe, were supplied by Dr. K. Yamashita of Kobe University and also by Dr. D. Smith of Virginia Polytechnic Institute and State University. Porcine thyroglobulin oligosaccharides (a biantennary fraction and also a mixture of tri- and tetraantennary fractions), human transferrin biantennary glycopeptide, human fibrinogen biantennary glycopeptide, and bovine fetuin triantennary oligosaccharide were the gifts of Dr. J. P. Carver of the University of Toronto.

Ovine submaxillary mucin and antifreeze glycoprotein carrying $\left[{ }^{14} \mathrm{C}\right] \mathrm{Neu}$ Ac $(\alpha 2-3)$ GalNAc and $\left[{ }^{14} \mathrm{C}\right]-$ Neu5Ac( $\alpha 2-6)$ GalNAc were provided by Dr. C. Paulson of the University of California at Los Angeles. Fetuin was purchased from Grand Island Biological Co. and orosomucoid was obtained from Dr. G. W. Jourdian of the University of Michigan. Asialo derivatives of both fetuin and orosomucoid were obtained by heating these glycoproteins in $0.1 \mathrm{~N}$ sulfuric acid for $1 \mathrm{~h}$ at $80^{\circ} \mathrm{C}$.

Labeling of oligosaccharides and glycopeptides. A small quantity of oligosaccharide (50-100 nmol) was reduced, at room temperature, with excess sodium borotritide (10-50 times excess on molar bases) for several hours or overnight. After the decomposition of excess reagent with acetic acid, the reaction product was purified by gel filtration on Biogel P-2. Glycopeptides were labeled by incubating with $\left[{ }^{14} \mathrm{C}\right]$ acetic anhydride for 1-2 $\mathrm{h}$ in $0.5 \% \mathrm{NaHCO}_{3}$. Labeled glycopeptides were also purified using the Biogel P-2 column.

Esterification and saponification of $\left[{ }^{3} H\right] N e u 5 A c(\alpha 2-$ 6)lactitol. ${ }^{8} \mathrm{H}$-labeled Neu5Ac( $\left.\alpha 2-6\right)$ lactitol was esterified using dimethylsulfoxide and methyl iodide ac- cording to the method of Handa et al. (16). The product was purified by gel filtration on Biogel P-2. Radioactive and Neu5Ac-positive fractions were collected and passed through a column of Dowex I-X8 (acetate form) to remove any unesterified material.

The ester was saponified by treatment with $0.1 \mathrm{~N}$ $\mathrm{NaOH}$ for $1 \mathrm{~h}$ at room temperature, and then passed successively through columns of Dowex $50 \mathrm{~W}-\times 8\left(\mathrm{H}^{+}\right.$ form) and Dowex I- $\times 8$ (acetate form). Free acid was obtained by eluting the latter column with $4 \mathrm{~N}$ acetic acid.

Affinity chromatography. Oligosaccharides, glycopeptides (1-10 nmol, in 30-100 $\mu$ l of distilled water or PBS), or glycoproteins (2 mg in $1 \mathrm{ml}$ PBS) were applied to the SNA-Sepharose column $(1.5 \times 14$ or $1 \times 52 \mathrm{~cm})$ followed by stepwise elution with PBS, PBS containing 50 or $100 \mathrm{~mm}$ lactose, and $20 \mathrm{mM}$ ethylenediamine. Gradient elution with 0-100 $\mathrm{mm}$ lactose also was used in some experiments. The flow rate was $10-15 \mathrm{ml} / \mathrm{h}$. The longer column was used for the experiment shown in Fig. 4; the shorter column was used for all other experiments. Elution was monitored either by measuring radioactivity or by absorption at $280 \mathrm{~nm}$. All experiments were performed at $4^{\circ} \mathrm{C}$.

\section{RESULTS}

Interaction of sialylated milk oligosaccharides with SNA-Sepharose. Various sialylated oligosaccharides obtained from human and bovine milk were labeled by reduction with sodium borotritide and the resulting $\left[{ }^{3} \mathrm{H}\right]$ oligosaccharide alditols were applied to the SNA-Sepharose column. The simplest oligosaccharide alcohol containing the $\operatorname{Neu} 5 \operatorname{Ac}(\alpha 2-6) \mathrm{Gal}$ sequenceNeu5Ac( $\alpha 2-6)$ lactitol-bound tightly to the SNA-Sepharose and was eluted with $50 \mathrm{mM}$ lactose (Fig. 1A). On the other hand, Neu5Ac $(\alpha 2-3)$ lactitol passed through the same column with the void volume (Fig. 1B). Analysis of commercial "Neu5Ac( $\alpha 2$ 6)lactose," after reduction with sodium borotritide, revealed the presence of both 2,3- and 2,6-isomers. The results of the analysis of other milk oligosaccharides, LSTa, LSTb, LSTc, and DSL (Fig. 2; structures are listed in Table I), showed that only LSTc, which carries one Neu5Ac( $\alpha 2-$ 6) Gal unit, bound to the SNA-Sepharose. Oligosaccharides which possess the Neu$5 \mathrm{Ac}(\alpha 2-3) \mathrm{Gal}$ or $\mathrm{Gal}(\beta 1-3)[\mathrm{Neu} \operatorname{Ac}(\alpha 2-6)]-$ GleNAc sequences did not bind to this column.

Interaction of glycopeptides and oligosaccharides obtained from glycoproteins with 


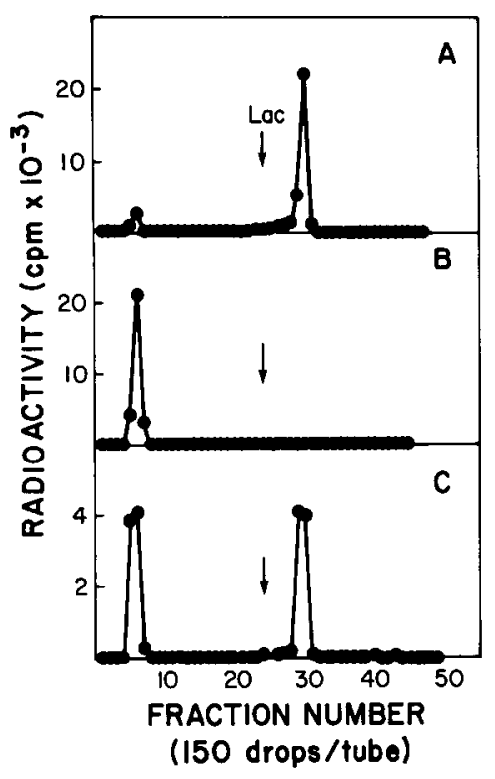

FIG. 1. Elution profile of sialyllactose on SNASepharose. (A) Pure Neu5Ac( $\alpha 2-6)$ lactose; (B) pure Neu5Ac( $\alpha 2-3)$ lactose; (C) commercial "Neu5Ac( $\alpha 2$ 6) lactose." Lac $=50 \mathrm{~mm}$ lactose in PBS.

SNA-Sepharose. A series of sialylated glycopeptides and oligosaccharides, which were labeled by acetylation of amino groups with $\left[{ }^{14} \mathrm{C}\right]$ acetic anhydride or by conversion to the corresponding alditol by reduction with sodium borotritide, also were applied to the SNA-Sepharose column. All compounds which contained at least one Neu5Ac $(\alpha 2-6) \mathrm{Gal}$ unit in the molecule bound firmly to the SNA-Sepharose (Fig. 3). Although the biantennary glycopeptides from human fibrinogen and porcine thyroglobulin, both of which carry a single Neu5Ac $(\alpha 2-6) \mathrm{Gal}$ unit, were eluted completely from the column with $50 \mathrm{~mm}$ lactose solution (Figs. 3A and B), the biantennary glycopeptide from human transferrin and the bovine fetuin triantennary oligosaccharide, both of which contain two units of this sequence, bound more strongly to the column and showed a retarded peak and also an additional peak which was eluted with $20 \mathrm{~mm}$ ethylenediamine (Figs. $3 \mathrm{C}$ and $\mathrm{D})$. These results suggested the possible use of the SNA-Sepharose column for the separation of glycopeptides/oligosaccharides based on their content of
Neu5Ac $(\alpha 2-6) \mathrm{Gal}$ units. Figure 4 shows the elution profile of a mixture of ${ }^{3} \mathrm{H}$-labeled porcine thyroglobulin biantennary oligosaccharide (containing one Neu5Ac( $\alpha 2$ 6)Gal) and ${ }^{14} \mathrm{C}$-labeled human transferrin biantennary glycopeptide (containing two Neu5Ac $(\alpha 2-6) \mathrm{Gal})$. The main fractions of the mixture of these compounds clearly were separated from each other by the use of a linear lactose gradient, although a shoulder on the peaks was observed for both of them. It is not clear whether these shoulder peaks suggest some heterogeneity of the samples used for this experiment or whether they originated from a "spillover" effect in ${ }^{3} \mathrm{H}$ and ${ }^{14} \mathrm{C}$ counting. A similar experiment consisting of a mixture of ${ }^{14} \mathrm{C}$-labeled human fibrinogen biantennary glycopeptide (one Neu5Ac $(\alpha 2-6) \mathrm{Gal}$ ) and ${ }^{3} \mathrm{H}$-labeled bovine fetuin triantennary oli-

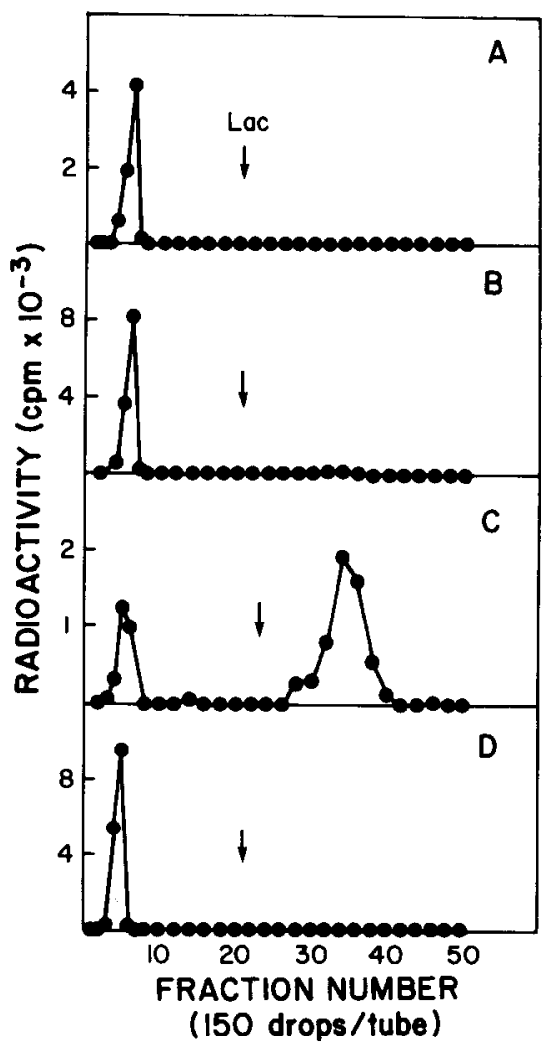

Fig. 2. Elution profile of milk oligosaccharides on SNA-Sepharose. (A) LSTa; (B) LSTb; (C) LSTc; (D) DSL. (See Table I for the structures.) 
SHIBUYA ET AL.

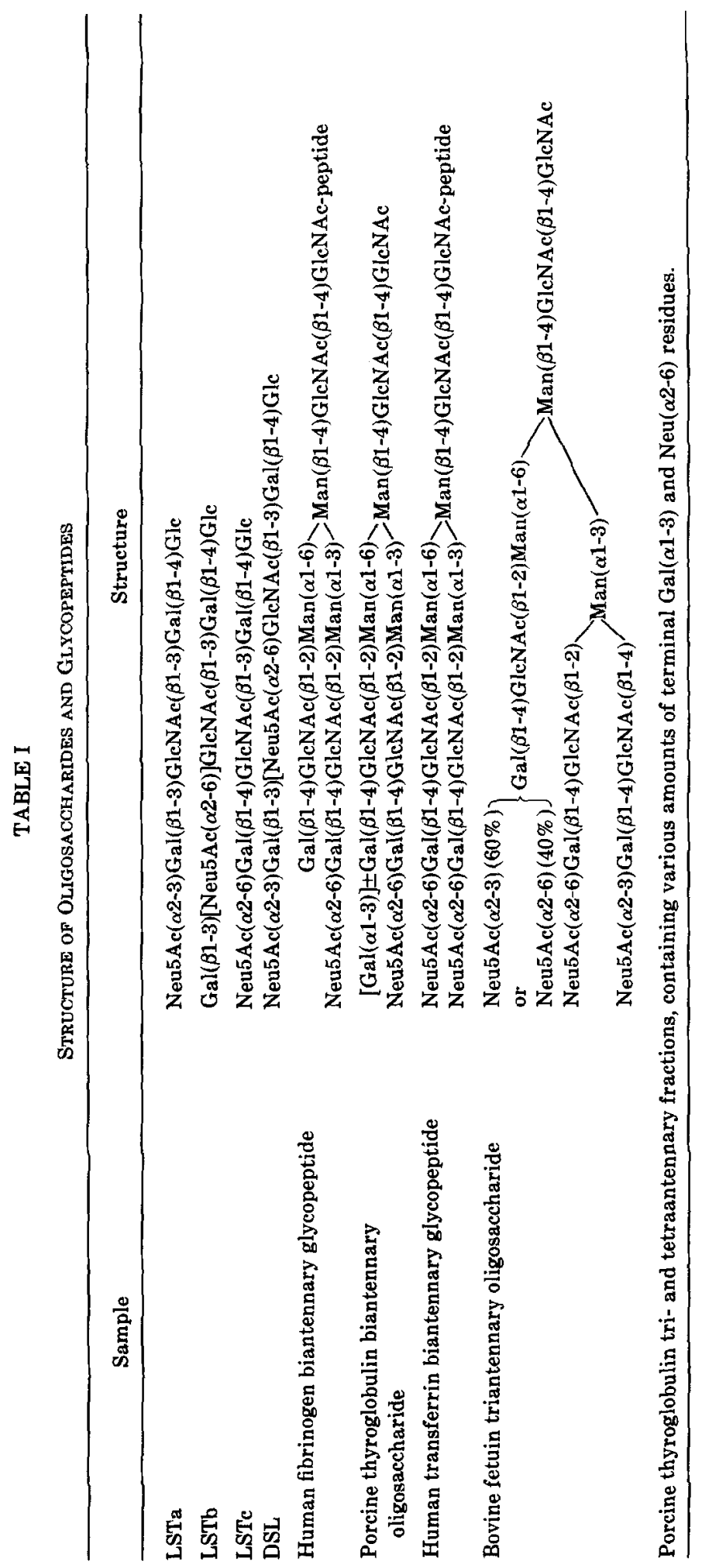




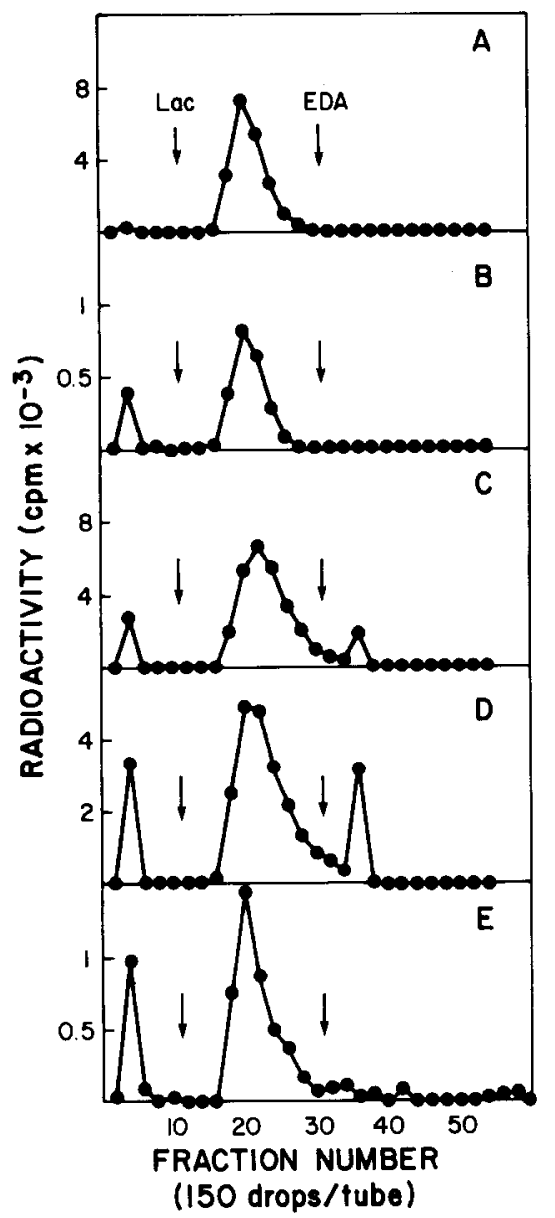

FIG. 3. Elution profile of oligosaccharides and glycopeptides obtained from glycoprotein on SNASepharose. (A) Human fibrinogen biantennary glycopeptide; (B) porcine thyroglobulin biantennary oligosaccharide; (C) human transferrin biantennary glycopeptide; (D) bovine fetuin triantennary oligosaccharide; (E) porcine thyroglobulin tri- and tetraantennary oligosaccharide. (See Table I for the structures.) $\mathrm{EDA}=20 \mathrm{mM}$ ethylene diamine.

gosaccharide (approximately $40 \%$ of it contains two Neu5Ac $(\alpha 2-6)$ Gal $)$ showed a marked retardation of only the fetuin triantennary peak (Fig. 5). We speculate that the first peak of the fetuin oligosaccharide represents the molecular species with one Neu5Ac $(\alpha 2-6) \mathrm{Gal}$ unit and the major portion of the retarded fraction contains two units of this sequence; further structural studies are necessary to support this speculation.
Interaction of glycoproteins with defined Neu5Ac-Gal/GalNAc sequences with SNASepharose. Ovine submaxillary mucin and antifreeze glycoprotein derivatives which contain defined carbohydrate chains synthesized in vitro using purified sialyl transferases $(17,18)$ were analyzed using the same column (Fig. 6). The ovine submaxillary mucin derivative which contains the Neu5Ac( $\alpha 2-6)$ GalNAc-Thr/Ser sequence bound tightly to the SNA-Sepharose, whereas the antifreeze glycoprotein carrying Neu5Ac $(\alpha 2-3) \operatorname{Gal}(\beta 1-3)$ GalNAc$\mathrm{Thr} /$ Ser did not bind (data not shown). Interestingly, the antifreeze glycoprotein which bears the Neu5Ac $(\alpha 2-6)$ GalNAc sequence with side chains attached to the $O$ 3 of the subterminal GalNAc did not bind to the column (Figs. $6 \mathrm{~B}$ and $\mathrm{C}$ ).

Contribution of the free carboxyl group of Neu5Ac to the interaction with SNA-Sepharose. We have already shown that the $O$ 3 and $O-4$ hydroxyl groups of Gal residues and the C-8 and/or C-9 hydroxyl groups of the glyceryl side chain of Neu5Ac are important for interaction of the Neu5Ac $(\alpha 2-$ 6)Gal unit with SNA (14). To determine the contribution of free carboxyl groups on the Neu5Ac residue, the methyl ester of $\left[{ }^{3} \mathrm{H}\right]$ Neu5Ac $(\alpha 2-6)$ lactitol was synthesized and applied to the SNA-Sepharose column. Neu5Ac $(\alpha 2-6)$ lactitol, which can bind to this column (Fig. 1), lost its ability to interact with the column after esterification of the carboxyl group of Neu5Ac (Fig. 7A). The ability of the esterified Neu5Ac to bind to the SNA-Sepharose was recovered by saponification of the ester (Fig. 7B), showing the importance of a frec carboxyl group on Neu5Ac for binding to SNA.

Application of SNA-Sepharose column to the analysis of glycoproteins. Two glycoproteins, bovine fetuin and orosomucoid, were applied separately to the immobilized SNA to determine the possible use of this technique for the analysis of microheterogeneity in the carbohydrate moiety of glycoproteins. As shown in Figs. 8A and B, portions of both fetuin and orosomucoid bound strongly to the SNA-Sepharose and these glycoproteins were separated into two fractions which were eluted with lactose and ethylenediamine, respectively, 


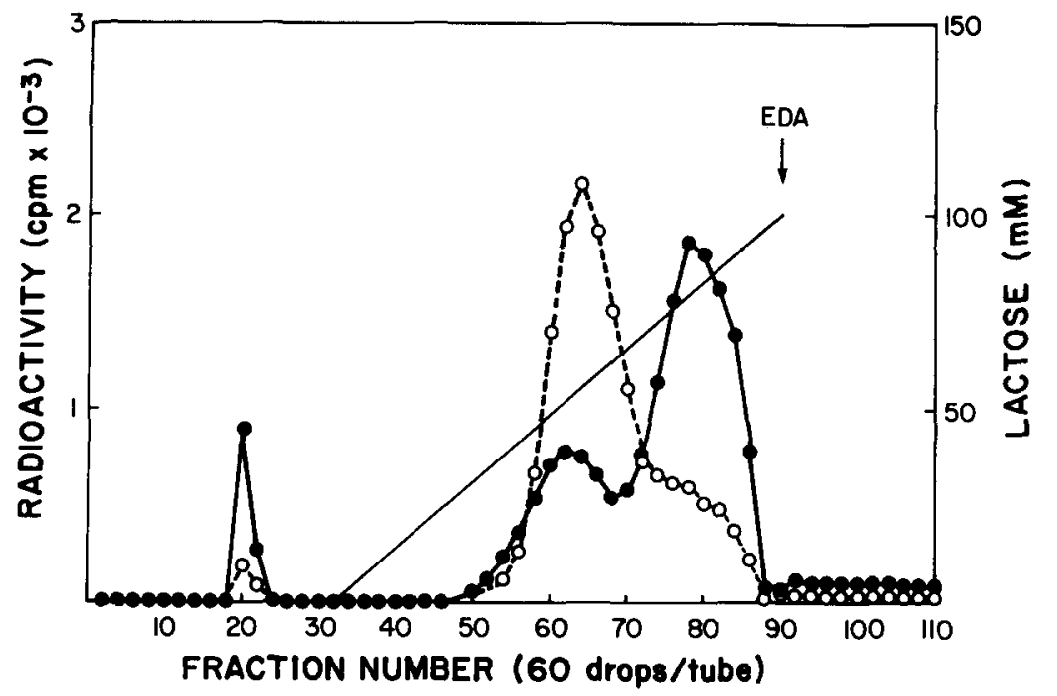

FIG. 4. Separation of porcine thyroglobulin biantennary oligosaccharide and human transferrin biantennary glycopeptide by the SNA-Sepharose column. ${ }^{3} \mathrm{H}$-labeled thyroglobulin oligosaccharide (O) and ${ }^{14} \mathrm{C}$-labeled transferrin glycopeptide (O) were mixed and applied to the SNA-Sepharose column. Elution was performed with a linear gradient of lactose, and then with $20 \mathrm{mM}$ ethylenediamine (EDA).

suggesting the presence of some types of microheterogeneity, most possibly due to the different density and distribution of Neu5Ac $(\alpha 2-6) \mathrm{Gal}$ sequences in these gly- coprotein molecules. Asialo derivatives of both glycoproteins passed through the column (only the elution profile of asialo fetuin is shown).

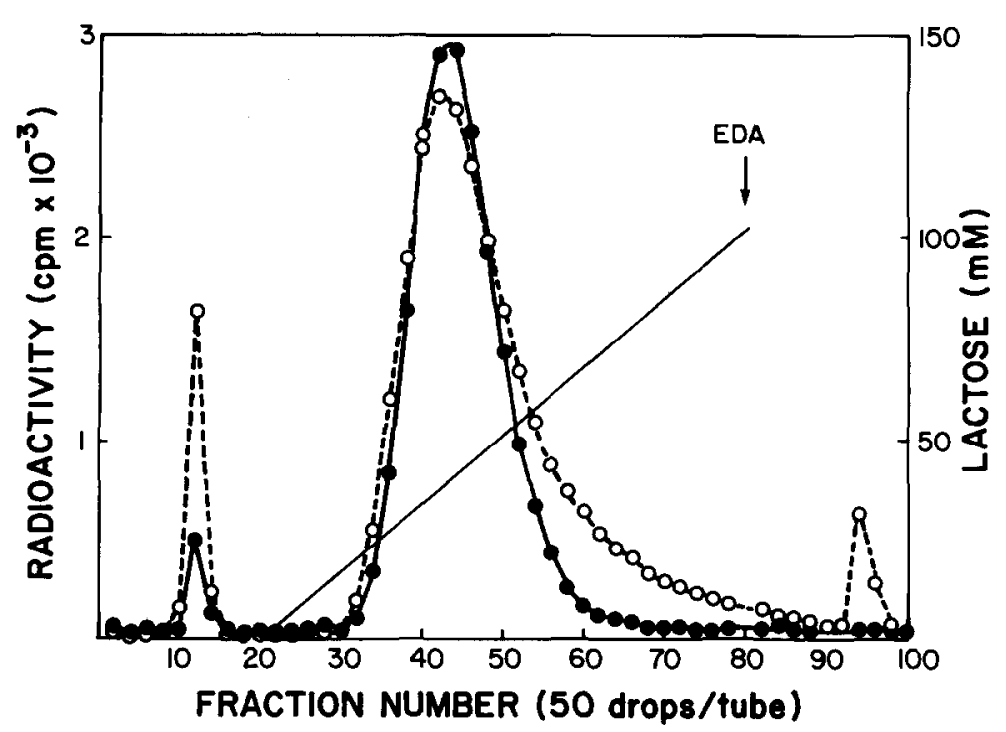

FIG. 5. Separation of human fibrinogen biantennary glycopeptide and porcine fetuin triantennary oligosaccharide by the SNA-Sepharose column. ${ }^{3} \mathrm{H}$-labeled fetuin oligosaccharide $(\mathrm{O})$ and ${ }^{14} \mathrm{C}$-labeled fibrinogen glycopeptide (๑) were mixed and applied to the SNA-Sepharose column. Elution was performed as described for Fig. 4. 


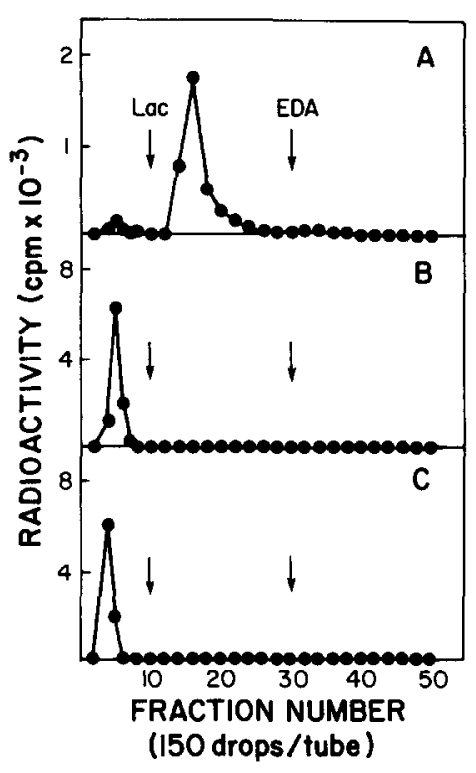

FIG. 6. Elution profile of glycoproteins containing Neu5Ac $(\alpha 2-6)$ GalNAc-Thr/Ser structure. (A) Ovine submaxillary mucin with Neu5Ac( $\alpha 2-6)$ GalNAc-Thr/ Ser; (B) antifreeze glycoprotein with Neu5Ac( $\alpha 2-$ 6)[Gal( $\beta 1-3)] \mathrm{GalNAc}-\mathrm{Thr} / \mathrm{Ser}$; (C) antifreeze glycoprotein with $\operatorname{Neu5Ac}(\alpha 2-6)[\operatorname{Neu} 5 \mathrm{Ac}(\alpha 2-3) \mathrm{Gal}(\beta 1-$ 3)]GalNAc-Thr/Ser. All the glycoproteins were first desialylated and then resialylated using specific sialyl transferases $(17,18)$ and $\left[{ }^{14} \mathrm{C}\right] \mathrm{Neu} 5 \mathrm{Ac}$.

\section{DISCUSSION}

The results described herein demonstrate that immobilized SNA can bind oli-

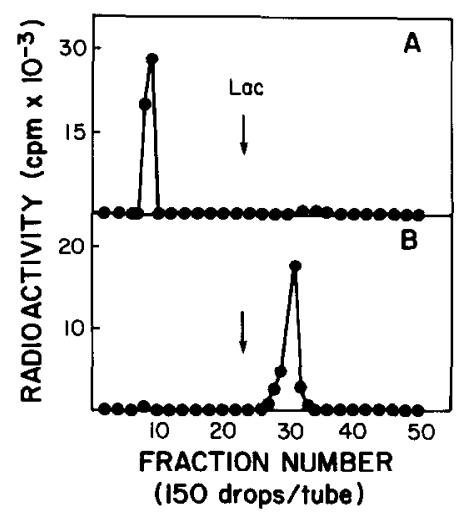

FIG. 7. Effect of the esterification of the carboxyl group of Neu5Ac on the interaction of $\left.{ }^{3} \mathrm{H}\right] \mathrm{Neu} 5 \mathrm{Ac}(\alpha 2-$ 6)lactitol with SNA-Sepharose. (A) Methyl ester of $\left[{ }^{3} \mathrm{H}\right.$ Neu5Ac $(\alpha 2-6)$ lactitol; (B) after saponification of methyl ester.

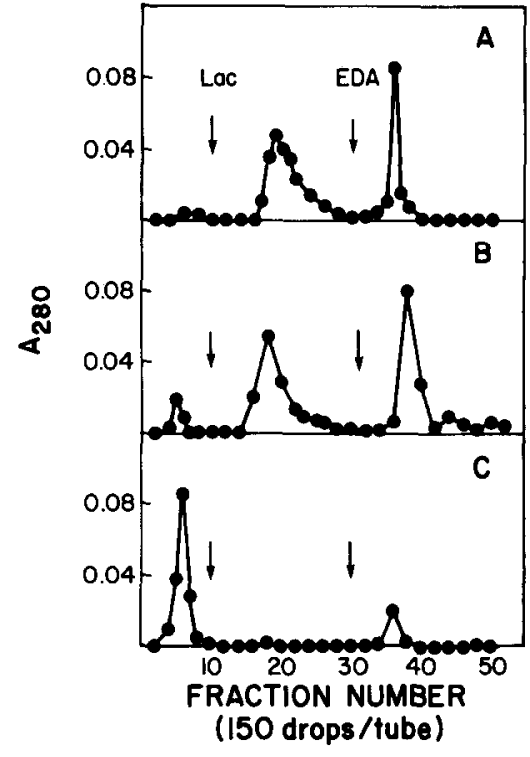

FIG. 8. Elution profile of orosomucoid, fetuin, and asialofetuin on SNA-Sepharose. (A) orosomucoid; (B) fetuin; (C) asialofetuin.

gosaccharides, glycopeptides, and glycoproteins which possess $\operatorname{Neu} 5 \mathrm{Ac}(\alpha 2-6) \mathrm{Gal} /$ GalNAc sequences, whereas those with Neu5Ac $(\alpha 2-3) \mathrm{Gal} / \mathrm{GalNAc}$ sequences are not bound. This important discovery has permitted us to separate clearly, molecular species which differ only in the type of linkages between terminal Neu5Ac and $\mathrm{Gal} / \mathrm{GalNAc}$ residues. The presence of one Neu5Ac $(\alpha 2-6)$ Gal unit was sufficient to permit the binding of oligosaccharides or glycopeptides to this column. It is noteworthy that even $\left[{ }^{3} \mathrm{H}\right] \mathrm{Neu} 5 \mathrm{Ac}(\alpha 2-6)$ lactitol, with an association constant $\left(K_{a}\right)$ for SNA $=3.9 \times 10^{5} \mathrm{M}^{-1}$, could bind to the SNASepharose, even though Baenziger and Fiete reported that glycopeptides with $K_{a}$ less than $4 \times 10^{6} \mathrm{M}^{-1}$ could not bind to Con A-Sepharose (19). The immobilized SNASepharose column is useful not only to separate the 2,6-linked isomers from 2,3-linked ones, but also is applicable for the fractionation of oligosaccharides, glycopeptides, and possibly glycoproteins based on their number of Neu5Ac $(\alpha 2-6) \mathrm{Gal}$ units (Figs. 4, 5, and 8).

The binding capacity of SNA-Sepharose estimated by an overloading experiment 
was approximately $2-3 \mathrm{nmol} / \mathrm{ml}$; in other words, $8-13 \%$ of the total carbohydrate binding sites of the immobilized SNA were active.

Branching of side chains from $\mathrm{O}-3$ of the subterminal GalNAc residue destroyed the capacity of glycoproteins to bind to the SNA-Sepharose (Fig. 6). This may be explained from the previous finding that the free hydroxyl group at $0-3$ and $O-4$ of Gal (possibly also GalNAc) are critical for the binding of SNA, though some steric effect also may contribute to this interference. The finding that the esterification of carboxyl group of Neu5Ac residue also abolished the binding ability of $\left[{ }^{3} \mathrm{H}\right] \mathrm{Neu} 5 \mathrm{Ac}(\alpha 2-$ 6)lactitol gave us further insight regarding the interaction of SNA with sialylated oligosaccharides. In addition to the $O-3$ and O-4 hydroxyl groups of $\mathrm{Gal}$ and the C-8, the C-9 portion of glyceryl side chain of Neu5Ac, free carboxyl groups of Neu5Ac also may be involved in the interaction.

From its unique ability to separate oligosaccharides, glycopeptides, or glycoproteins based on the type of linkage between Neu5Ac and Gal/GalNAc, SNA should be a very useful tool for the detection, isolation, and characterization of these compounds.

\section{REFERENCES}

1. Corfield, A. P., ANd Schauer, R. (1982) in Sialic Acids: Metabolism and Function (Schauer, R., Ed.), pp. 5-51, Springer-Verlag, Vienna/New York.

2. Smit, H., GaAstra, W., Kamerling, J. P., VlieGENTHART, J. F. G., AND DEGRAAF, F. K. (1984) Infect. Immun. 46, 578-584.
3. Paulson, J. C., Sadler, J. E., AND HILl, R. L. (1979) J. Biol Chem. 254, 2120-2124.

4. Rogers, G. N., ANd Paulson, J. C. (1983) Virology 127, 361-373.

5. Suzuki, Y., Matsunaga, M., and Matsumoto, M. (1985) J. Biol Chem. 260, 1362-1365.

6. GlaSGOW, L. R., AND HILL, R. L. (1980) Infect. Immun. 30, 353-361.

7. Loomes, L. M., Uemura, K., Childs, R. A., PaULSON, J. C., Rogers, G. N., ScudDeR, P. R., MiCHALsKi, J. C., Hounsell, E. F., TAYloR-RoBinSON, D., AND FeIZI, T. (1984) Nature (London) 307, 560-563.

8. Roelcke, D., Pruzanski, W., Ebert, W., Romer, W., Fisher, E., LENHARD, V., AND RAUTERBERG, E. (1980) Blood 55, 677-681.

9. Hakomori, S., Patterson, C. M., Nudelman, E., AND SekiguChi, K. (1983) J. Biol. Chem. 258, 11819-11822.

10. Schauer, R., Shukla, A. K., Schroder, C., AND Muller, E. (1984) Pure Appl. Chem. 56, 907921.

11. FINNE, J., AND KRUsius, T. (1982) in Methods in Enzymology (Ginsburg, V., Eds.), Vol. 83, pp. 269-277, Academic Press, New York.

12. SMith, D. F., Zopf, D. A., AND GinsBuRG, V. (1978) in Methods in Enzymology (Ginsburg, V., Ed.), Vol. 50, pp. 221-226, Academic Press, New York.

13. Broekaert, W. F., Nsimba-Lubaki, M., Peeters, B., AND Peumans, W. J. (1984) Biochem. J. 221, 163-169.

14. Shibuya, N., Goldstein, I. J., Brakkaert, W. F., Nsimba-Lubaki, M., Peeters, B., and Peumans, W. J. (1987) J. Biol. Chem, in press.

15. March, S. C., Parakh, I., and Cuatrecasas, P. (1974) Anal Biochem. 60, 149-152.

16. HANDA, S., AND NakamURA, K. (1984) $J$. Biochem. 95, 1323-1329.

17. Sadler, J. E., Paulson, J. C., AND HILl, R. L. (1979) J. Biol Chem. 254, 2112-2119.

18. Sadler, J. E., Rearick, J. I., Paulson, J. C., AND HILL, R. L. (1979) J. Biol. Chem. 254, 4434-4443.

19. Baenziger, J. U., AND Fiete, D. (1979) J. Biol. Chem. 254, 2400-2407. 